\title{
Optimal Contract for Wind Power in Day-Ahead Electricity Markets
}

\author{
Desmond W. H. Cai ${ }^{1} \quad$ Sachin Adlakha ${ }^{2} \quad$ K. Mani Chandy
}

\begin{abstract}
The growth of wind energy production poses several challenges in its integration in current electric power systems. In this work, we study how a wind power producer can bid optimally in existing electricity markets. We derive optimal contract size and expected profit for a wind producer under arbitrary penalty function and generation costs. A key feature of our analysis is to allow for the wind producer to strategically withhold production once the day ahead contract is signed. Such strategic behavior is detrimental to the smooth functioning of electricity markets. We show that under simple conditions on the offered price and marginal imbalance penalty, a risk neutral profit maximizing wind power producer will produce as much as wind power is available (up to its contract size).
\end{abstract}

\section{Introduction}

The insatiable appetite for energy as well as concerns for global warming have led to greater emphasis on research and development of "cleaner" sources of energy. Several states have enacted renewable portfolio standards that call for utilities to expand their renewable portfolios [1]. Wind energy is expected to play a major role in achieving these goals. Due to rapid installation, low maintenance and low operational costs, there has been explosive growth in the installed wind capacity over the last few years [2]. However, due to its inherent variable nature, integrating wind power plants into current electric systems pose several challenges to both wind power producers as well as system operators [3, 4]. To overcome these challenges and to enable wind penetration, we need to better understand the impact of uncertain production in electricity markets.

Current electricity markets consist of primarily two modes of operation - bilateral trading and competitive

This paper is supported in part by NSF NET-SE award 0911041.

${ }^{1}$ D. W. H. Cai is with the Department of Electrical Engineering, California Institute of Technology, Pasadena, CA, USA. wccai@caltech.edu

${ }^{2} \mathrm{~S}$. Adlakha is with the Center for Mathematics of Information, California Institute of Technology, Pasadena, CA, USA. adlakha@caltech.edu

${ }^{3} \mathrm{~K}$. M. Chandy is with the Department of Computer Science, California Institute of Technology, Pasadena, CA, USA. mani@cs.caltech.edu electricity pools $[5,6,7]$. In the former mode, generators and loads negotiate a bilateral contract to transfer certain amount of electricity at a future date. In competitive electricity pools, buyers and sellers participate in a single market cleared by a third party - called the Independent Service Operator (ISO). These markets typically consist of a day-ahead (DA) forward market as well as a real-time (RT) market. In a DA market, each producer (buyer) submits a bid (offer) to the ISO to supply (purchase) electricity for the next day. The ISO then uses this data to compute the aggregate supply and demand function and determine the market clearing price. All producers that submitted bids below the market clearing price are scheduled and hence are obligated to dispatch their power the following day. Furthermore, all schedules submitted to the DA market are cleared (i.e., financial transactions are completed) before the beginning of the RT market $[8,9]$.

Given the current market structure, a wind power producer that is scheduled, is required to dispatch power when asked by the system operator. Because the ability of a producer depends on the current wind strength, the inherent uncertainty makes it harder for the ISO to rely on the wind power. From the viewpoint of the wind power producer, if the available wind power is less than what it was scheduled for, the wind power producer incurs an imbalance penalty. This penalty is derived because the wind power producer is required to purchase the shortfall from the RT market, which typically has higher prices. Thus, given the uncertainty in its production, a wind power producer may withhold its capacity from the DA market. This in turn could result in lower wind penetration.

Integration of wind power into power systems has attracted considerable interest in recent years [10]. The impact of wind uncertainty on electricity markets was studied in [11, 12]. As argued in [13], the value of wind energy decreases as the volatility of wind increases. To incorporate the dynamics and uncertainty of wind in electricity markets, the authors in [14] provide a general economic equilibrium model that incorporates salient features of electricity markets.

In this paper, we focus on a scenario in which a wind power producer participates in a DA market. We study the problem of optimal contract size from the viewpoint of a wind power producer. We assume that wind power 
constitutes a small fraction of the total market and hence the wind power producer is price-taking. Furthermore, we assume that the real time market is exogenous and hence we assume a functional form for the imbalance penalty. We derive the optimal DA contract for the wind power producer under general imbalance penalty, general generation costs, and arbitrary wind power distributions. The problem of optimal contracting for wind power producers was also studied in [15], where the authors used linear programming to solve for short term optimal trading strategy for wind power producers. The work that is closely related to our analysis is [16]. In that work, the authors derived optimal bid amount and optimal expected profits for wind power producers. Compared to that work, we consider a more general imbalance penalty model as well as production costs.

An important feature of our analysis is to allow for strategic behavior of the wind power producer. In other words, we allow the wind power producer to curtail its production (even if sufficient wind power is available) once the DA contract has been signed. We show that under simple conditions, a wind power producer will have no incentive to deviate from its contract as long as sufficient wind power is available. This truthful production is important since it allows for smooth functioning of markets. We also study the role of increased uncertainty on the optimal contract size as well as optimal profits for wind power producers. We show that under increased uncertainty (defined in the usual first order stochastic dominance sense), the optimal contract size and optimal profits decrease. The results derived in this paper are for arbitrary distribution of wind, operating costs and imbalance penalties subject to only convexity and monotonicity assumptions; our results can thus be applied to a wide range of scenarios (e.g., to study other renewable energy sources).

\section{Model}

We consider a single DA electricity market in which producers offer contracts to supply electricity. Contracts offered in a DA market typically consist of piecewise constant power levels over fixed time intervals (which are usually 1 hour intervals). Without the presence of any storage capacity, a contract offered in a single time interval is independent of the contract offered in another time interval. Thus, for the purposes of this paper, we will focus our attention on a single time interval denoted by $I$. Each producer would offer a contract $c$ (in MW), which is the amount of power it will deliver over this time interval. The producer is paid $p$ (in $\$ / \mathrm{MW}$ ) for this supplied power; we assume that the producers are price-takers. In typical markets, the contracts are settled much before the interval in which the power is supposed to be delivered. Once the contract is signed and settled, the producer is paid the amount $\$ p c$. We assume that producers are risk neutral and profit maximizers.

For a wind power producer, the amount of electricity that it can generate will depend on the available wind at that time. Let us denote by $W$ the total wind (in terms of MW of its production capability) that is available at the beginning of the time interval. We assume that $W$ is a continuous random variable distributed over the interval $[0, \bar{w}]$ with a probability density function denoted by $f$ and the corresponding distribution function $F$. We assume that $f$ does not have any jump at zero, i.e., $F(0)=0$. We also assume that the total wind available stays constant over the desired time interval $I$.

At the beginning of the time interval $I$, the producer produces a quantity $q \mathrm{MW}$ of power. This production amount is obviously dependent on the contract size as well as the available wind. The producer incurs an operational cost of $\$ g(q)$ to produce this quantity. Note that a producer could decide to produce a quantity that is less than both its contract size as well as the available wind. To discourage the producers from over-committing in the DA market, an imbalance penalty of $\$ h\left((c-q)^{+}\right)$ is charged to the producer. Here $(x)^{+}=\max \{x, 0\}$; in other words, only the shortfall from the contract is penalized. Given a contract size $c$, and a chosen production level $q$, the producer's profit is given by

$$
\Pi(c, q)=p c-h\left((c-q)^{+}\right)-g(q)
$$

Once the contract is signed, the producer will choose its production quantity to maximize its profit. This production quantity cannot be greater than the wind power available to the producer. Let us define the optimal production as

$$
q^{*}(c, w)=\arg \max _{0 \leq q \leq w} \Pi(c, q)
$$

where $w$ is the actual wind available to the wind power producer.

In a DA market, the actual realization of the wind power available is not known. Thus, a producer will choose a DA contract to maximize his expected profit. Given a distribution $F$ on the available wind power, let us define the expected DA profit of a producer as

$$
\begin{aligned}
\bar{\Pi}_{F}(c) & =\mathbb{E}\left[\max _{0 \leq q \leq W} \Pi(c, q) \mid W \sim F\right], \\
& =\mathbb{E}\left[\Pi\left(c, q^{*}(c, W)\right) \mid W \sim F\right] .
\end{aligned}
$$

The DA profit of a producer is based on the assumption that once a contract is decided, the producer chooses an optimum production level $q^{*}(c, W)$ that depends on its chosen contract size $c$ and the actual wind power available $W$. The optimal DA contract of a producer is then given by

$$
c_{F}^{*}=\arg \max _{c \geq 0} \bar{\Pi}_{F}(c) .
$$


For notational brevity, we will often drop the subscript $F$ wherever the distributional dependence is unambiguous. We make the following assumption on the cost of production as well as the imbalance penalty.

Assumption 1. The cost of production $g$ and the imbalance function $h$ are increasing ${ }^{1}$, twice differentiable, convex functions with $g(0)=0$ and $h(0)=0$. Furthermore, we assume that the offered price $p$ is greater than the marginal cost of production at zero, i.e., $p \geq g^{\prime}(0)$.

The above assumption is fairly mild. The cost of production is typically an increasing and convex function of the quantity produced. Furthermore, the imbalance penalty should increase as the deviation from the contract is increased. The requirement that $p \geq g^{\prime}(0)$ ensures that the offered price is large enough that the producer would have some incentive to produce. Assumption 1 is kept throughout the remainder of the paper ${ }^{2}$.

\section{Main Results}

\subsection{Optimal Production}

We begin by looking at the optimal production level for a producer, once a contract has been signed in the DA market. The key question we would like to answer is if the producer has an incentive to deviate from its contract and produce a quantity that is lower than its contract size as well as the total wind power available. Intuitively speaking, if the cost of production is much higher than the imbalance penalty, the producer might curtail its production to lower its cost and pay the imbalance penalty. Such deviations from the DA contracts are detrimental to proper functioning of electricity markets. The next lemma shows that for any contract size, as long as the marginal imbalance penalty is high enough, any risk-neutral profit-maximizing producer would produce as much as wind power is available up to its contract size.

Lemma 1. Given any contract $c$, if $h^{\prime}(0) \geq g^{\prime}(c)$, then for any realization of available wind $w$, the producer has no incentive to deviate from its contract. Thus, the optimal production is given by $q^{*}(c, w)=\min \{c, w\}$.

Proof. We consider two different cases.

Case 1: Let us first consider the case when $w \leq c$. For all $q \leq w \leq c$, we have $h\left((c-q)^{+}\right)=h(c-q)$ and

\footnotetext{
${ }^{1}$ A function $f: \mathbb{R} \rightarrow \mathbb{R}$ is said to be increasing if for every $x, y \in \mathbb{R}$, $x>y$, we have $f(x) \geq f(y)$. We say the function is strictly increasing if the inequality is strict.

${ }^{2}$ Given Assumption 1, the profit in equation (1) is a continuous function of the production quantity. Thus, the maximization problem in equation (2) is well defined. Furthermore, if the expected profit in equation (3) is always increasing, we define the optimal contract $c^{*}=\infty$
}

thus we have $\Pi(c, q)=p c-h(c-q)-g(q)$. Note that

$$
\begin{aligned}
\frac{\partial \Pi(c, q)}{\partial q} & =h^{\prime}(c-q)-g^{\prime}(q), \\
& \geq h^{\prime}(c-q)-g^{\prime}(c) \\
& \geq h^{\prime}(0)-g^{\prime}(c) .
\end{aligned}
$$

Here the first inequality follows from the fact that the cost of production is a convex function (Assumption 1) and hence $g^{\prime}(q)$ is an increasing function for $0 \leq q \leq w \leq$ $c$. The second inequality follows because the imbalance penalty is also a convex function (Assumption 1), thus $h^{\prime}(c-q)$ is a decreasing function for all $0 \leq q \leq c$. So if $h^{\prime}(0) \geq g^{\prime}(c)$, then $\Pi(c, q)$ is an increasing function for all $0 \leq q \leq w$ and hence $q^{*}(c, w)=w$.

Case 2: Now consider the case when $c \leq w$. Note that

$$
\begin{aligned}
\max _{0 \leq q \leq w} \Pi(c, q) & =\max \left\{\max _{0 \leq q \leq c} \Pi(c, q), \max _{c \leq q \leq w} \Pi(c, q)\right\}, \\
& =\max \left\{\Pi(c, c), \max _{c \leq q \leq w} \Pi(c, q)\right\},
\end{aligned}
$$

where the last equality follows from Case 1 by taking $w=$ c. Note that for all $c \leq q \leq w$, we have $h\left((c-q)^{+}\right)=0$ and thus we have

$$
\max _{c \leq q \leq w} \Pi(c, q)=\max _{c \leq q \leq w}\{p c-g(q)\}=\Pi(c, c) .
$$

Here the last equality follows from the fact that $g$ is an increasing function (Assumption 1). Hence, if $c \leq w$, then

$$
q^{*}(c, w)=\arg \max _{0 \leq q \leq w} \Pi(c, q)=c .
$$

This proves the lemma.

The above lemma states that a producer would try to produce as much as wind power is available up to its contract size and would not deviate from its contract if the lowest marginal imbalance penalty is greater than its marginal cost of production at the contract size. The intuitive explanation is that a producer is better-off fulfilling its contract if the savings from reducing production are less than the imbalance penalty.

\subsection{Optimal Day-Ahead Contract}

The previous lemma ensures that given any contract size and any wind resource available, a profit-maximizing producer would supply as much as wind power is available up to its contract size as long as the marginal imbalance penalty is high enough. Note that $g$ is a convex function and hence $g^{\prime}$ is an increasing function. It is possible that beyond a certain contract size, the conditions in Lemma 1 may not hold. Thus, at that contract size, it is possible that the optimal production for a producer is less than both its contract size and the wind power available. The key question is whether a risk-neutral profit-maximizing 
producer will ever choose such a contract size in a DA market. In this section, we show that the answer to this question is no as long as the marginal imbalance penalty is strictly higher than the offered price. We formalize this in the following assumption.

Assumption 2. We assume that the lowest marginal imbalance penalty is strictly higher than the offered price. That is, $h^{\prime}(0)>p$.

Note that this assumption implies that $h$ is a strictly increasing function. This assumption is kept throughout the remainder of the paper. We will show below that under this assumption, a profit-maximizing risk-neutral producer will choose a DA contract size such that under that contract size its optimum production level is to produce as much as wind power is available up to its contract size. To prove this, we first make the following definition.

Definition 1. Let $\hat{c}$ be defined as follows:

$$
\hat{c}=\arg \max _{x \geq 0}\{p x-g(x)\} .
$$

Using the first order conditions, it is easy to see that $\hat{c}$ satisfies $p=g^{\prime}(\hat{c})$. Since the function $p x-g(x)$ is concave, the first order conditions are also sufficient. If the above maximization problem has multiple optimal solutions, we define $\hat{c}$ to be the largest of those solutions. From Assumption 1, we know that $p \geq g^{\prime}(0)$, thus $\hat{c}$ is well-defined. Now, if $p>g^{\prime}(x)$ for all $x \geq 0$, then $p x-g(x)$ is always increasing. In this case, we define $\hat{c}=\infty$. For the remainder of this section, we make the following assumption.

Assumption 3. We assume that there exists a finite $\hat{c}$ satisfying $\hat{c}=\arg \max _{x \geq 0}\{p x-g(x)\}$.

The above assumption is only made for technical convenience. In Section 4.1, we study the case where $\hat{c}$ is infinite. We show that all of our results continue to hold.

The next lemma shows that given any distribution on the wind production, the optimal contract under that distribution is always less than $\hat{c}$.

Lemma 2. Given a distribution $F$ on the wind production, the optimal DA contract satisfies $c_{F}^{*} \leq \hat{c}$.

Proof. From Assumption 2, Assumption 3, and Definition 1 , we have for all $c \leq \hat{c}$,

$$
\begin{aligned}
h^{\prime}(0) & >p \\
& =g^{\prime}(\hat{c}) \geq g^{\prime}(c),
\end{aligned}
$$

where the last inequality follows since $g$ is a convex function (Assumption 1). From equation (4) and equation (3), we have

$$
\mathbb{E}\left[\Pi\left(c^{*}, q^{*}\left(c^{*}, W\right)\right)\right] \geq \mathbb{E}\left[\Pi\left(c, q^{*}(c, W)\right)\right] \forall c .
$$

Here the expectation is taken over the random variable $W$ with distribution function $F$.

Suppose there exist a distribution $F$ such that $c^{*}>\hat{c}$. To prove the lemma, we show that if $c^{*}>\hat{c}$, then given any wind power $w$, we have

$$
\Pi\left(c^{*}, q^{*}\left(c^{*}, w\right)\right)<\Pi\left(\hat{c}, q^{*}(\hat{c}, w)\right) .
$$

This will contradict equation (6) and will prove the lemma. Note that

$$
\Pi\left(c^{*}, q^{*}\left(c^{*}, w\right)\right)=\max _{0 \leq q \leq w} \Pi\left(c^{*}, q\right),
$$

and

$$
\Pi\left(\hat{c}, q^{*}(\hat{c}, w)\right)=\max _{0 \leq q \leq w} \Pi(\hat{c}, q),
$$

where $\Pi(c, q)$ is given by equation (1). To prove that if $c^{*}>\hat{c}$, equation (7) holds for all $w$, we analyze three different cases.

Case 1. Consider the case where $w \leq \hat{c}<c^{*}$. Then, we have

$$
\begin{aligned}
\Pi\left(c^{*}, q^{*}\left(c^{*}, w\right)\right) & =\max _{0 \leq q \leq w}\left\{p c^{*}-h\left(\left(c^{*}-q\right)^{+}\right)-g(q)\right\} \\
& =\max _{0 \leq q \leq w}\left\{p c^{*}-h\left(c^{*}-q\right)-g(q)\right\} .
\end{aligned}
$$

We next show that the argument in the above maximization problem is always increasing. Note that for all $q \leq w \leq \hat{c}<c^{*}$, we have

$$
\frac{\partial \Pi\left(c^{*}, q\right)}{\partial q}=h^{\prime}\left(c^{*}-q\right)-g^{\prime}(q)>h^{\prime}\left(c^{*}-q\right)-h^{\prime}(0) \geq 0 .
$$

Here the first inequality follows from equation (5) and the last inequality follows from the fact that $h$ is a convex function. Thus, we have $\Pi\left(c^{*}, q^{*}\left(c^{*}, w\right)\right)=\Pi\left(c^{*}, w\right)$. Similarly, we can show that $\Pi\left(\hat{c}, q^{*}(\hat{c}, w)\right)=\Pi(\hat{c}, w)$. Note that for all $\hat{c} \leq c \leq c^{*}$ and $w \leq \hat{c}<c^{*}$, we have

$$
\Pi(c, w)=p c-h(c-w)-g(w),
$$

and

$$
\frac{\partial \Pi(c, w)}{\partial c}=p-h^{\prime}(c-w) \geq p-h^{\prime}(0)>0 .
$$

The first inequality follows from the fact that $h$ is a convex function and the last inequality follows from Assumption 2. Thus, we have that $\Pi(c, w)$ is strictly increasing in $c$ for all $\hat{c} \leq c \leq c^{*}$ and $w \leq \hat{c}<c^{*}$. Hence, for all $w \leq \hat{c}<c^{*}$, we have $\Pi\left(c^{*}, w\right)<\Pi(\hat{c}, w)$.

Case 2: Now consider the case where $\hat{c} \leq w<c^{*}$. We have

$$
\begin{aligned}
\Pi\left(c^{*}, q^{*}\left(c^{*}, w\right)\right) & =\max _{0 \leq q \leq w}\left\{p c^{*}-h\left(c^{*}-q\right)-g(q)\right\} \\
& <\max _{0 \leq q \leq w}\left\{p c^{*}-p\left(c^{*}-q\right)-g(q)\right\} \\
& =\max _{0 \leq q \leq w}\{p q-g(q)\} \\
& \leq p \hat{c}-g(\hat{c}) .
\end{aligned}
$$


The last inequality follows from the definition of $\hat{c}$. For the first inequality, notice that for all $q \leq w<c^{*}$, we have $c^{*}-q>0$. Furthermore, $h$ is a convex function, so $h\left(c^{*}-q\right) \geq h^{\prime}(0)\left(c^{*}-q\right)>p\left(c^{*}-q\right)$. Here, the strict inequality follows since we have $h^{\prime}(0)>p$ and $c^{*}-q>0$. Now consider

$$
\begin{aligned}
\Pi\left(\hat{c}, q^{*}(\hat{c}, w)\right) & =\max _{0 \leq q \leq w} \Pi(\hat{c}, q), \\
& =\max \left\{\max _{0 \leq q \leq \hat{c}} \Pi(\hat{c}, q), \max _{\hat{c} \leq q \leq w} \Pi(\hat{c}, q)\right\}, \\
& =\max \left\{\Pi(\hat{c}, \hat{c}), \max _{\hat{c} \leq q \leq w} \Pi(\hat{c}, q)\right\} .
\end{aligned}
$$

Here the last equality follows from the analysis in Case 1. Note that for all $\hat{c} \leq q$, the imbalance penalty is zero, and hence $\max _{\hat{c} \leq q \leq w} \Pi(\hat{c}, q)=p \hat{c}-g(\hat{c})$. This implies that $\Pi\left(\hat{c}, q^{*}(\hat{c}, w)\right)=p \hat{c}-g(\hat{c})$. Thus, for all $\hat{c} \leq w<c^{*}$, we have $\Pi\left(c^{*}, q^{*}\left(c^{*}, w\right)\right)<\Pi\left(\hat{c}, q^{*}(\hat{c}, w)\right)$.

Case 3. For the case when $c^{*} \leq w$, we have

$$
\begin{aligned}
\Pi\left(c^{*}, q^{*}\left(c^{*}, w\right)\right) & =\max _{0 \leq q \leq w} \Pi\left(c^{*}, q\right), \\
& =\max \left\{\max _{0 \leq q<c^{*}} \Pi\left(c^{*}, q\right), \max _{c^{*} \leq q \leq w} \Pi\left(c^{*}, q\right)\right\}, \\
& =\max \left\{\max _{0 \leq q<c^{*}} \Pi\left(c^{*}, q\right), p c^{*}-g\left(c^{*}\right)\right\} .
\end{aligned}
$$

Here, the last equality follows by noting that for $c^{*} \leq q$ the imbalance penalty is zero. From Case 2, we have that $\max _{0 \leq q<c^{*}} \Pi\left(c^{*}, q\right)<p \hat{c}-g(\hat{c})$. From the definition of $\hat{c}$, we must have that $\Pi\left(c^{*}, q^{*}\left(c^{*}, w\right)\right)<p \hat{c}-g(\hat{c})$. Following the steps similar to equation (10), it is easy to verify that $\Pi\left(\hat{c}, q^{*}(\hat{c}, w)\right)=p \hat{c}-g(\hat{c})$.

Thus for all possible wind power realizations, if $c^{*}>\hat{c}$, we have $\Pi\left(c^{*}, q^{*}\left(c^{*}, w\right)\right)<\Pi\left(\hat{c}, q^{*}(\hat{c}, w)\right)$ which violates equation (6). This proves the lemma.

The following theorem is the main result of this paper.

Theorem 1. Given any distribution $F$ on the wind power, the optimal DA contract for a producer is given by the solution to the following equation.

$$
p=\int_{0}^{c^{*}} h^{\prime}\left(c^{*}-w\right) f(w) d w+g^{\prime}\left(c^{*}\right)\left(1-F\left(c^{*}\right)\right) .
$$

Furthermore, given any realization of the actual wind $w$, the optimal production for a producer is $q^{*}\left(c^{*}, w\right)=$ $\min \left\{c^{*}, w\right\}$.

Proof. From Assumption 2 and Assumption 3, we have $h^{\prime}(0)>p=g^{\prime}(\hat{c})$. Since $g$ is a convex function, we have $g^{\prime}(\hat{c}) \geq g^{\prime}(c)$ for all $c \leq \hat{c}$. Thus, $h^{\prime}(0)>g^{\prime}(c)$ for all $c \leq \hat{c}$. From Lemma 2, we know that for any possible wind distribution $F, c^{*} \leq \hat{c}$, where $c^{*}$ is the optimal DA contract under that distribution. Using Lemma 1, we get that $q^{*}\left(c^{*}, w\right)=\min \left\{c^{*}, w\right\}$. From equation (4), we know that the optimal contract is given by

$$
\begin{aligned}
c^{*} & =\arg \max _{0 \leq c} \mathbb{E}\left[\Pi\left(c, q^{*}(c, W)\right)\right], \\
& =\arg \max _{0 \leq c \leq \hat{c}} \mathbb{E}\left[\Pi\left(c, q^{*}(c, W)\right)\right], \\
& =\arg \max _{0 \leq c \leq \hat{c}} \mathbb{E}[\Pi(c, \min \{c, W\})] .
\end{aligned}
$$

Here the second equality follows since $c^{*} \leq \hat{c}$ and the last equality follows from Lemma 1 . We have

$$
\begin{gathered}
\mathbb{E}\left[\Pi\left(c, q^{*}(c, W)\right)\right]=p c-\int_{0}^{c}(h(c-w)+g(w)) f(w) d w \\
-g(c)(1-F(c)) .
\end{gathered}
$$

It is easy to verify that the right hand side of the above equation is a concave function for all $c \leq \hat{c}$. Using the first order conditions we get the desired result.

\subsection{Impact of Uncertainty}

In this section we study the impact of uncertainty on the optimal DA contract of the producers. Intuitively speaking, we expect that as the uncertainty in the wind distribution increases, the producers would offer a lower DA contract. We measure uncertainty as the probability that the wind power exceeds a certain quantity. The higher this probability, the lower the uncertainty in the wind power. We formalize this notion using the first order stochastic dominance ordering on the set of distributions.

Definition 2. We say a distribution $F^{\prime}$ first order stochastically dominates another distribution $F$ (written as $\left.F^{\prime} \succeq_{1} F\right)$ if $F^{\prime}(x) \leq F(x)$ for all $x \in[0, \bar{w}]$.

Let $f^{\prime}$ be the density associated with $F^{\prime}$ and $f$ be the density associated with the distribution $F$. It is straightforward to show that if $F^{\prime} \succeq_{1} F$, then

$$
\int_{0}^{\bar{w}} u(w) f^{\prime}(w) d w \geq \int_{0}^{\bar{w}} u(w) f(w) d w
$$

for all increasing bounded functions $u$ on $[0, \bar{w}][17]$.

Theorem 2. Suppose $F^{\prime} \succeq_{1} F$. Let $c_{F^{\prime}}^{*}$ be the optimal DA contract under distribution $F^{\prime}$ and let $c_{F}^{*}$ be the optimal DA contract under distribution F. Then,

1. $c_{F^{\prime}}^{*} \geq c_{F}^{*}$.

2. $\bar{\Pi}\left(c_{F^{\prime}}^{*}\right) \geq \bar{\Pi}\left(c_{F}^{*}\right)$.

Proof. Let us define $\Lambda_{F}(c)$ as

$$
\begin{aligned}
& \Lambda_{F}(c)=\int_{0}^{c} h^{\prime}(c-w) f(w) d w+g^{\prime}(c)(1-F(c)), \\
& =h^{\prime}(0) F(c)-h^{\prime}(c) F(0)+\int_{0}^{c} h^{\prime \prime}(c-w) F(w) d w+ \\
& \quad g^{\prime}(c)(1-F(c)), \\
& =g^{\prime}(c)+F(c)\left(h^{\prime}(0)-g^{\prime}(c)\right)+\int_{0}^{c} h^{\prime \prime}(c-w) F(w) d w .
\end{aligned}
$$


From Theorem 1, we know that the optimal contract is given by $p=\Lambda_{F}\left(c_{F}^{*}\right)$. From Lemma 2 , it suffices to consider the function $\Lambda_{F}(c)$ for all $c \leq \hat{c}$. From Assumption 2, Assumption 3, and the definition of $\hat{c}$, we have $h^{\prime}(0)>g^{\prime}(c)$ for all $c \leq \hat{c}$. It is easy to verify that

$$
\begin{array}{r}
\frac{d \Lambda_{F}(c)}{d c}=\int_{0}^{c} h^{\prime \prime}(c-w) f(w) d w \\
+g^{\prime \prime}(c)(1-F(c)) \\
+f(c)\left(h^{\prime}(0)-g^{\prime}(c)\right) .
\end{array}
$$

Thus, for all $c \leq \hat{c}, \Lambda_{F}(c)$ is an increasing function.

Since $F^{\prime} \succeq_{1} F$, we have $F^{\prime}(c) \leq F(c)$ for all $c$. From Assumption 1, we have that $h$ is a convex function and hence $h^{\prime \prime} \geq 0$. Thus, we have $\Lambda_{F^{\prime}}(c) \leq \Lambda_{F}(c)$ for all $c \leq$ $\hat{c}$. Thus, we have $p=\Lambda_{F}\left(c_{F}^{*}\right) \geq \Lambda_{F^{\prime}}\left(c_{F}^{*}\right)$. Since $\Lambda_{F^{\prime}}(c)$ is an increasing function, the point at which $\Lambda_{F^{\prime}}(c)$ crosses $p$ is greater than $c_{F}^{*}$. This proves the first part.

To prove the second part, we note that

$$
\begin{aligned}
& \bar{\Pi}_{F}(c) \\
& =p c-\int_{0}^{c}(h(c-w)+g(w)) f(w) d w-g(c)(1-F(c)), \\
& =p c-(h(0)+g(c)) F(c) \\
& -\int_{0}^{c}\left(h^{\prime}(c-w)-g^{\prime}(w)\right) F(w) d w-g(c)(1-F(c)), \\
& =p c-g(c)-\int_{0}^{c}\left(h^{\prime}(c-w)-g^{\prime}(w)\right) F(w) d w .
\end{aligned}
$$

Let us again restrict our attention to $c \leq \hat{c}$. Then, we have $h^{\prime}(c-w)>g^{\prime}(w)$ for all $0 \leq w \leq c$. Thus for $F^{\prime} \succeq_{1} F$, we have $\bar{\Pi}_{F^{\prime}}(c) \geq \bar{\Pi}_{F}(c)$ for all $c \leq \hat{c}$. Since $c^{*} \leq \hat{c}$, we have $\bar{\Pi}_{F^{\prime}}\left(c_{F^{\prime}}^{*}\right) \geq \bar{\Pi}_{F^{\prime}}\left(c_{F}^{*}\right) \geq \bar{\Pi}_{F}\left(c_{F}^{*}\right)$. Here the first inequality follows because $c_{F^{\prime}}^{*}$ maximizes $\bar{\Pi}_{F^{\prime}}(c)$. This proves the theorem.

For $F^{\prime} \succeq_{1} F$, the probability that at least $w \mathrm{MW}$ of wind power is higher under $F^{\prime}$ than under the distribution $F$. Clearly the producer is better off under distribution $F^{\prime}$ than it is under distribution $F$.

\section{Discussion}

\subsection{Relaxing Assumption 3}

The results in the previous section were proved under the assumption that $\hat{c}$ is finite. This was mainly done for technical convenience. In this subsection, we show that Assumption 3 can be relaxed. Observe that if $\hat{c}$ is infinite, then $p c-g(c)$ is unbounded so we have that $p \geq g^{\prime}(c)$ for all $c \geq 0$. By Assumption 2, it then follows that $h^{\prime}(0)>g^{\prime}(c)$ for all $c \geq 0$. From Lemma 1, the optimal production for the producer at any contract $c$ is always $q^{*}(c, w)=\min \{c, w\}$. Hence, the optimal contract is given by

$$
c^{*}=\arg \max _{0 \leq c} \mathbb{E}[\Pi(c, \min \{c, W\})]
$$

It is easy to check that the first order condition for this maximization problem is given by (11), and hence Theorem 1 holds.

To prove Theorem 2 , note that since $h^{\prime}(0)>g^{\prime}(c)$ for all $c, \Lambda_{F}(c)$ as defined in equation (12) is an increasing function for all $c$. Similarly, given any $c, \bar{\Pi}_{F}(c)$ is an increasing function of the distribution $F$ (where the set of distributions is ordered in the FOSD sense). The rest of the proof follows.

\subsection{Linear Cost and Linear Imbalance Penalty}

In this section we look at a special case of linear cost and linear imbalance penalty. This case was considered in [16] and we show that in this case the above results imply those of $[16]$. We let

$$
\begin{aligned}
g(q) & =\alpha q, \\
h\left((c-q)^{+}\right) & =\lambda(c-q)^{+},
\end{aligned}
$$

where $\alpha$ and $\lambda$ are the marginal cost of production and marginal imbalance penalty. The assumptions in the paper reduce to $\lambda>p \geq \alpha$ which ensure that the marginal penalty is higher than the offered price which in turn is higher than the marginal cost of production. From Theorem 1, we get that the optimal day ahead contract is given by

$$
c^{*}=F^{-1}\left(\frac{p-\alpha}{\lambda-\alpha}\right) .
$$

In other words, $c^{*}$ is the $((p-\alpha) /(\lambda-\alpha))^{t h}$-quantile of $F$. The optimal expected profit can be written as

$$
\bar{\Pi}\left(c^{*}\right)=(\lambda-\alpha) \int_{0}^{F\left(c^{*}\right)} F^{-1}(\theta) d \theta .
$$

In other words, the expected profit is proportional to the area to the left of the distribution curve $F$ from 0 to $F\left(c^{*}\right)$.

\subsection{Revisiting Assumption 2}

As stated in Assumption 2, we require that the lowest marginal imbalance penalty is higher than the offered price. As we see in this section, if this assumption is violated, a risk-neutral profit-maximizing producer can profit from over contracting. As an example we consider a scenario where the imbalance penalty is a linear function given by $h\left((c-q)^{+}\right)=\lambda(c-q)^{+}$. For a given day ahead contract, the expected profit of a producer in a day ahead market is given by

$$
\begin{gathered}
\mathbb{E}\left[\max _{0 \leq q \leq W} \Pi(c, q)\right]=\mathbb{E}\left[\max _{0 \leq q \leq W}\left(p c-\lambda(c-q)^{+}-g(q)\right)\right] \\
=c(p-\lambda)+\mathbb{E}\left[\max _{0 \leq q \leq W}\{\lambda \min \{c, q\}-g(q)\}\right]
\end{gathered}
$$

where we have used the fact that $(c-q)^{+}=c-\min \{c, q\}$. Now suppose that a producer chooses a contract size $c \geq$ 
$\bar{w}$. Then, clearly its production will be $q \leq c$ since the maximum wind power is bounded. The producer's DA expected profit can then be written as

$\mathbb{E}\left[\max _{0 \leq q \leq W} \Pi(c, q)\right]=c(p-\lambda)+\mathbb{E}\left[\max _{0 \leq q \leq W}\{\lambda q-g(q)\}\right]$,

which is an increasing function of $c$. For $\lambda<p$, the producer can make arbitrarily large profit by choosing large contract sizes. If $\lambda=p$, the producer is completely indifferent to the size of its contract. Thus Assumption 2 is important to ensure that producers' offered contracts are consistent with their production.

\section{Conclusions}

In this paper, we study optimal contract size for a wind power producer participating in a day-ahead electricity market. For a simple scenario where the wind power producer is price-taking, we derive optimal contract size and optimal expected profit under general production cost and imbalance penalty functions. Allowing for wind power producers to be strategic, we show that as long as the marginal imbalance penalty is higher than the offered price, the wind power producer will produce as much wind power as is available in real-time. This shows that the ISO can schedule wind power producers without worrying about their strategic behavior.

A key extension to our model would be to allow the imbalance penalty to be an outcome of a real-time market. In such scenarios, the wind power producer's expected day-ahead profit would depend on the outcome of the real-time market. This would substantially change its strategic behavior and it is possible that the optimal decision for a wind power producer may be to offer zero contract in a day-ahead market. Such behavior would not bode well for large scale wind integration without the presence of subsidies. Investigating such market outcomes is the subject of our current investigation.

\section{Acknowledgements}

We thank Prof. Steven Low and Prof. Adam Weirman of California Institute of Technology for helpful discussions.

\section{References}

[1] R. Wiser, "Renewable Portfolio Standards in the United States - A Status Report with Data Through 2007," Tech. Rep. LBNL-154E, Lawrence Berkeley National Laboratory, 2008.

[2] G. W. E. Council, "Global Wind 2009 Report," tech. rep., Renewable Energy House, Brussels, Belgium, 2009.

[3] G. E. Consulting, "Intermittency analysis project: appendix B. Impact of intermittent generation on operation of california power grid," Tech. Rep. CEC-500-2007081-APB, GE Energy Consulting, 2007.
[4] G. Energy, "Western Wind and Solar Integration Study," tech. rep., National Renewable Energy Laboratory (NREL), Golden, CO., 2010.

[5] D. S. Kirschen and G. Strbac, Fundamentals of Power System Economics. Wiley, 2004.

[6] S. Stoft, Power System Economics: Designing Markets for Electricity. Wiley-IEEE Press, 2002.

[7] C. Harris, Electricity Markets: Pricing, Structures and Economics (The Wiley Finance Series). Wiley, 2006.

[8] "California Independent System Operator Corporation Fifth Replacement FERC Electric Tariff, CAISO, 2011," [Online] Available: www.caiso.com/2b18/2b1872f312430.pdf.

[9] "California Independent System Operator DayAhead Market, CAISO, 2010," [Online] Available: www.caiso.com/docs/2000/07/19/200007191535315040 .pdf.

[10] E. A. DeMeo, G. A. Jordan, C. Kalich, J. King, M. R. Milligan, C. Murley, B. Oakleaf, and M. J. Schuerger, "Accommodating wind's natural behavior," IEEE Power and Energy Magazine, vol. 5, no. 6, pp. 5967, 2007.

[11] J. Cox, "Impact of Intermittency: How Wind Variability Could Change the Shape of the British and Irish Electricity Markets," 2009. [Online] Available: www.uwig.org/ImpactofIntermittency.pdf.

[12] M. Negrete-Pincetic, G. Wang, A. Kowli, and H. PulgarPainemal, "Emerging issues due to the integration of wind power in competitive electricity markets," in Power and Energy Conference at Illinois (PECI), pp. 45-50, 2010.

[13] S. Meyn, M. Negrete-Pincetic, G. Wang, A. Kowli, and E. Shafieepoorfard, "The value of volatile resources in electricity markets," in Proc. of the 49th Conf. on Dec. and Control, pp. 4921-4926, 2010.

[14] G. Wang, A. Kowli, M. Negrete-Pincetic, E. Shafieepoorfard, and S. Meyn, "A control theorists perspective on dynamic competitive equilibria in electricity markets," in Proc. 18th World Congress of the International Federation of Automatic Control (IFAC), 2011.

[15] J. M. Morales, A. J. Conejo, and J. Pérez-Ruiz, "Shortterm trading for a wind power producer," IEEE Transactions on Power Systems, vol. 25, no. 1, pp. 554-564, 2010.

[16] E. Bitar, R. Rajagopal, P. Khargonekar, K. Poolla, and P. Varaiya, "Bringing wind energy to market," IEEE Transactions on Power Systems. submitted for publication.

[17] M. Shaked and J. G. Shanthikumar, Stochastic Orders and their Applications. Associated Press, 1994. 